\title{
Transverse instability of energy-exchanging counterpropagating waves in photorefractive media
}

\author{
M. Saffman, A. A. Zozulya, and D. Z. Anderson \\ Department of Physics and Joint Institute for Laboratory Astrophysics, University of Colorado, Boulder, Colorado, 80309-0440
}

Received October 25, 1993; revised manuscript received March 2, 1994

\begin{abstract}
The transverse instability of counterpropagating waves in photorefractive media that is due to the formation of reflection gratings is studied. We obtain new solutions for the instability threshold that account for the reflection-grating-mediated transfer of energy between the pump waves. We find that the instability threshold is minimized for equal-intensity pump beams inside the medium, corresponding to externally supplied beams of unequal intensity when there is energy coupling between the pump beams. A couplingconstant-times-interaction-length product of at least 1.1 when the coupling constant is purely real, or at least 6.3 when the coupling constant is purely imaginary, is sufficient for observation of transverse instabilities. Comparison with the physically available photorefractive coupling constant leads to the conclusion that a variety of commonly available materials should be suitable for the observation of transverse instabilities. In addition, new results for the instability threshold of a reflection-grating four-wave mixing oscillation are obtained that include the effects of direct coupling between the pump waves.
\end{abstract}

\section{INTRODUCTION}

Transverse modulational instability of two counterpropagating beams in nonlinear optical media has been observed in atomic vapors, ${ }^{1-3}$ liquid crystals, ${ }^{4,5}$ and photorefractive crystals. ${ }^{6}$ The instability manifests itself in the far field by the appearance of conical rings, pairs of spots, or an array of spots, sometimes with hexagonal symmetry. The transverse intensity modulation in the near field has the characteristic spatial scale $l_{s}=2 \pi / \theta_{c} k_{0}$, where $\theta_{c}$ is the small angle between the generated satellite beams and the primary beams. The angle $\theta_{c}$ at which the threshold of the absolute instability is minimum may be found for all the above materials by a linearized analysis of the equations of motion. ${ }^{7-12}$ On the other hand, the nature of the nonlinear stage of the instability, the details of the resulting patterns, and their spatial and temporal stability appear to vary widely among different materials. In atomic vapors cones, pairs of spots, and hexagonal or other arrays of spots may all be observed, depending on the frequency and the intensity of the primary beams. In liquid crystals the instability leads to a stable pattern, sometimes of hexagonal symmetry, whereas the recent observations in photorefractive media indicate a somewhat different behavior. Here the hexagonal pattern is well defined but temporally and spatially unstable, such that it appears as a cone in a time-averaged recording. The cones observed in atomic vapors may also be due to unstable hexagons, although time-resolved data are not available at present. There is some reason to suspect this cause since, as was shown in Ref. 3, the cone may be collapsed into a hexagon by injection of a weak beam at $\theta_{c}$. The intrinsically slow dynamics of photorefractive media simplify time-resolved measurements, rendering photorefractives well suited to further experimental investigation of these phenomena.

A recent analysis of the modulational instability in photorefractive media that is due to the formation of trans- mission gratings showed that the strong incoherent scattering (fanning) characteristic of photorefractive media considerably raises the otherwise quite low instability threshold. ${ }^{12}$ The fanning may be effectively suppressed by the use of narrow pump beams, although this is not appropriate in the case of transmission gratings, since there will then be few modulation fringes across the primary beams. It was shown by Honda ${ }^{6}$ that this problem may be circumvented when the photorefractive crystal is oriented such that the instability is due to the formation of reflection gratings. In this case the grating vector $\mathbf{k}_{g}=2 \mathbf{k}_{0}$ is parallel to the primary beams, which results in many modulation fringes, even with narrow beams. In instantaneous Kerr-type media the presence of reflection gratings does not significantly complicate the analysis, since the resulting phase transfer between the primary beams may be scaled out of the problem. However, the formation of reflection gratings in photorefractive media generally leads to strong energy coupling between the primary beams. Indeed, in the experiments of Honda that used photorefractive $\mathrm{KNbO}_{3}$ the intensity-coupling coefficient between the counterpropagating pump waves was measured to be $12.5 \mathrm{~cm}^{-1} .6$ The strong energy coupling and the fact that the photorefractive coupling constant is complex render existing treatments of this problem inapplicable, necessitating the new analysis presented below.

The instability threshold in the geometry shown in Fig. 1 depends on several characteristic quantities: the photorefractive coupling constant $\gamma$, which is complex; the angle $\theta$ between the primary and satellite beams; the intensity ratio of the counterpropagating beams, given by $q=I_{B} / I_{F} ;$ and also the boundary conditions. The characteristic angle between the primary and the satellite beams is such that the phase lag that is due to diffraction is compensated for by nonlinear phase transfer between the primary and the satellite beams. The instability threshold is therefore lowest when the coupling constant $\gamma$ is purely real, which maximizes the phase transfer between the 


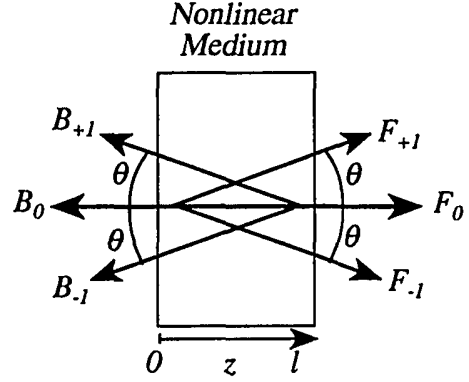

(a)

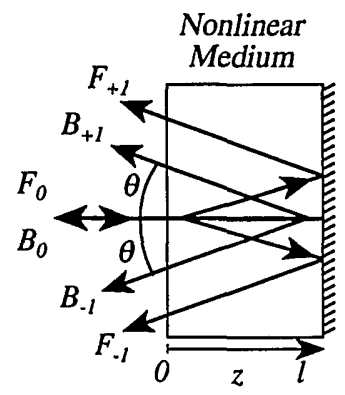

(b)

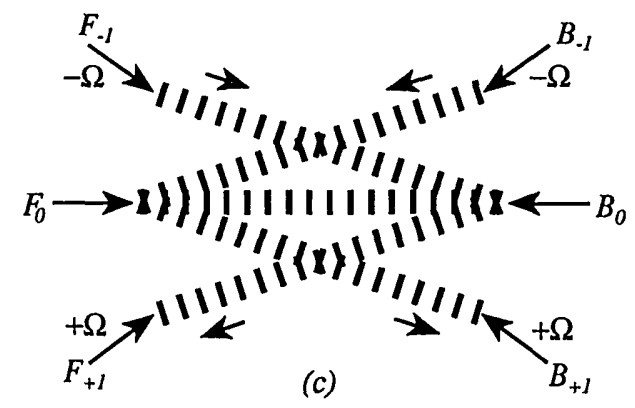

Fig. 1. Geometry of the optical interaction: (a) Two externally supplied counterpropagating primary beams. (b) One external beam and mirror boundary conditions at $z=l$. (c) Three reflection gratings couple the beam pairs $\left(F_{0}, B_{0}\right),\left[\left(F_{0}, B_{-1}\right),\left(F_{+1}\right.\right.$, $\left.\left.B_{0}\right)\right]$ and $\left[\left(F_{0}, B_{+1}\right),\left(F_{-1}, B_{0}\right)\right]$. The directions of motion of the moving gratings are indicated by the short arrows.

beams. However, in the reflection geometry studied here $\gamma$ is almost purely imaginary. In this case the sidebands will typically detune such that the coupling constant governing the interaction of the primary beams with the sidebands acquires a larger real part and hence increased phase transfer. The sidebands may also be detuned even when $\gamma$ is purely real if the pump beams have unequal intensities. This phenomenon is well known from the theory of photorefractive four-wave mixing. ${ }^{13}$

The equations of motion and the resulting dispersion relation giving the instability threshold for the case of real $\gamma$ are presented in Sections 2 and 3 below. The analytical expressions are derived allowing for arbitrary values of $q$, although numerical evaluation of the dispersion relation reveals that the threshold is lowest for $q=1$, in agreement with the transmission-grating case. ${ }^{12}$ The solutions with real $\gamma$ serve as a point of comparison for the general case of complex $\gamma$ given in Section 4. When the imaginary part of $\gamma$ is nonzero then $q=q(z)$, which complicates the equations of motion. Analytical solutions may be found by choosing particular boundary condi- tions such that $q$ is constant even though there is strong energy coupling between the counterpropagating beams. In Fig. 1(a) the nonlinear medium is illuminated with counterpropagating primary beams of prescribed intensity. In Fig. 1(b) one end of the nonlinear medium is coated with a perfectly reflecting surface. Both types of boundary condition lead to a constant beam-intensity ratio throughout the photorefractive medium. Numerical solutions of the dispersion relations for the case of imaginary $\gamma$ show that the instability threshold is increased several times over compared with the case of real $\gamma$.

In Section 5 we consider the relative suitability of particular photorefractive media for the experimental observation of spatial instabilities by accounting for the dependence of the coupling constant on material parameters. We find that the necessary coupling constant is equal to the available coupling constant within a factor of 2 in centimeter-sized samples of $\mathrm{BaTiO}_{3}, \mathrm{LiNbO}_{3}$, or $\mathrm{KNbO}_{3}$. On the other hand, large variations in the coupling coefficient may be found in different samples of what are nominally the same photorefractive material. The implication is that it may be necessary to handpick good samples for the observation of transverse instabilities.

\section{EQUATIONS OF MOTION}

Consider two strong plane waves $F_{0} \exp \left[i\left(k_{0} z-\omega_{0} t\right)\right]$ and $B_{0} \exp \left[i\left(-k_{0} z-\omega_{0} t\right)\right]$ counterpropagating in a nonlinear medium in the geometry of Fig. 1(a). We assume that these waves interact directly with each other through the formation of a reflection grating proportional to $F_{0} B_{0}^{*} \exp \left(i k_{g} z\right)$ with the grating vector $k_{g}=2 k_{0}$. The resulting redistribution of intensity and phase between the counterpropagating beams is described by the equations

$$
\begin{aligned}
\frac{\mathrm{d} F_{0}}{\mathrm{~d} z} & =i \gamma_{p} \frac{q}{1+q} F_{0}, \\
\frac{\mathrm{d} B_{0}}{\mathrm{~d} z} & =-i \gamma_{p}^{*} \frac{1}{1+q} B_{0}, \\
q(z) & =\frac{\left|B_{0}(z)\right|^{2}}{\left|F_{0}(z)\right|^{2}}
\end{aligned}
$$

In writing Eqs. (1a) and (1b) we have assumed that passive losses that are due to absorption are negligible. The coupling constant $\gamma_{p}$ is generally complex and is a function of material parameters, the grating vector $\mathbf{k}_{g}$ and a possible frequency shift $\Omega$ between the interacting beams. The frequency-degenerate counterpropagating primary beams interact through $\gamma_{p} \equiv \gamma\left(\mathbf{k}_{g}=2 k_{0} \hat{z}\right.$, $\Omega=0$ ).

The counterpropagating beams may be absolutely unstable to the appearance of sidebands propagating at a small angle with respect to the main beams. Referring to Fig. 1(c), consider a weak probe wave $F_{+1} \exp \left[i\left(k_{0} z+\right.\right.$ $\left.\left.\mathbf{k}_{\perp} \cdot \mathbf{r}_{\perp}\right)-i\left(\omega_{0}+\Omega\right) t\right]$ with $\left|\mathbf{k}_{\perp}\right| \ll \mathbf{k}_{0},|\Omega| \ll \omega_{0}$. It interacts with the counterpropagating strong pump wave $B_{0}$, resulting in a nonlinear change in the refractive index of the medium proportional to $F_{+1} B_{0}{ }^{*} \exp \left[i\left(2 k_{0} z+\right.\right.$ $\left.\left.\mathbf{k}_{\perp} \cdot \mathbf{r}_{\perp}\right)-i \Omega t\right]$ plus its complex conjugate. Scattering of pump wave $B_{0}$ off the index grating supports $F_{+1}$, and 
scattering of pump wave $F_{0}$ off the conjugate part supports $B_{-1}$. Similarly, the weak sideband $F_{-1} \exp \left[i\left(k_{0} z-\right.\right.$ $\left.\left.\mathbf{k}_{\perp} \cdot \mathbf{r}_{\perp}\right)-i\left(\omega_{0}-\Omega\right) t\right]$ interacts with the counterpropagating strong pump $B_{0}$, and scattering of the pump waves off the refractive-index perturbation supports the sidebands $F_{-1}$ and $B_{+1}$. Transmission gratings that are due to the interaction of pump wave $F_{0}$ with its sidebands $F_{+1}$ and $F_{-1}$ and of pump wave $B_{0}$ with its sidebands $B_{+1}$ and $B_{-1}$ will in general also be formed. We assume in what follows that the interaction geometry is chosen such that transmission gratings are weak and may be neglected. This is an excellent approximation in photorefractive media when the pump beams propagate along the crystal$\operatorname{lographic} c$ axis. Our previous analysis ${ }^{12}$ considered the opposite situation, in which transmission gratings but not reflection gratings are formed.

The full set of interacting waves is written in the form

$$
\begin{aligned}
F\left(\mathbf{r}_{\perp}, z, t\right)= & F_{0}(z)\left\{1+F_{+1} \exp \left[i\left(\mathbf{k}_{\perp} \cdot \mathbf{r}_{\perp}-\Omega t\right)\right]\right. \\
& \left.+F_{-1} \exp \left[i\left(-\mathbf{k}_{\perp} \cdot \mathbf{r}_{\perp}+\Omega t\right)\right]\right\} \\
& \times \exp \left[i\left(k_{0} z-\omega_{0} t\right)\right], \\
B\left(\mathbf{r}_{\perp}, z, t\right)= & B_{0}(z)\left\{1+B_{+1} \exp \left[i\left(\mathbf{k}_{\perp} \cdot \mathbf{r}_{\perp}-\Omega t\right)\right]\right. \\
& \left.+B_{-1} \exp \left[i\left(-\mathbf{k}_{\perp} \cdot \mathbf{r}_{\perp}+\Omega t\right)\right]\right\} \\
& \times \exp \left[i\left(-k_{0} z-\omega_{0} t\right)\right] .
\end{aligned}
$$

Linearizing the equations of motion in the amplitudes of the sidebands results in the set

$$
\begin{aligned}
\left(\frac{\mathrm{d}}{\mathrm{d} z}+i k_{d}\right) F_{+1}= & i \frac{q}{1+q}\left[\left(\chi+\gamma_{0}-\gamma_{p}\right) F_{+1}+\gamma_{p} B_{+1}\right. \\
& \left.+\chi+\gamma_{0} B_{-1}^{*}\right] \\
\left(\frac{\mathrm{d}}{\mathrm{d} z}-i k_{d}\right) F_{-1}^{*}= & -i \frac{q}{1+q}\left[\left(\chi_{-}^{*} \gamma_{0}^{*}-\gamma_{p}^{*}\right) F_{-1}^{*}\right. \\
& \left.+\chi_{-}^{*} \gamma_{0}^{*} B_{+1}+\gamma_{p}^{*} B_{-1}^{*}\right] \\
\left(\frac{\mathrm{d}}{\mathrm{d} z}-i k_{d}\right) B_{+1}= & -i \frac{1}{1+q}\left[\gamma_{p}^{*} F_{+1}+\chi_{-}^{*} \gamma_{0}^{*} F_{-1}^{*}\right. \\
& \left.+\left(\chi{ }^{*} \gamma_{0}^{*}-\gamma_{p}^{*}\right) B_{+1}\right] \\
\left(\frac{\mathrm{d}}{\mathrm{d} z}+i k_{d}\right) B_{-1}^{*}= & i \frac{1}{1+q}\left[\chi+\gamma_{0} F_{+1}+\gamma_{p} F_{-1}^{*}\right. \\
& \left.+\left(\chi+\gamma_{0}-\gamma_{p}\right) B_{-1}^{*}\right]
\end{aligned}
$$

The characteristic diffraction wave vector is given by $k_{d}=k_{\perp}{ }^{2} / 2 k_{0}$; the frequency-shifted sidebands interact with the primary beams through $\gamma\left(2 \mathbf{k}_{0} \pm \mathbf{k}_{\perp}, \pm \Omega\right)=$ $\chi_{ \pm} \gamma\left(2 \mathbf{k}_{0} \pm \mathbf{k}_{\perp}, 0\right)$, where $\chi_{ \pm}=1 /(1 \mp i \Omega \tau)$; and, since $\left|\mathbf{k}_{\perp}\right| \ll \mathbf{k}_{0}, \gamma\left(2 \mathbf{k}_{0} \pm \mathbf{k}_{\perp}, 0\right)$ has been approximated by $\gamma\left(2 \mathbf{k}_{0}, 0\right) \equiv \gamma_{0}$. We have allowed for different pump-pump $\left(\gamma_{p}\right)$ and pump-sideband $\left(\gamma_{0}\right)$ coupling constants in Eqs. (3). These may arise if the pump beams have different polarization states or have imperfect temporal coherence, which would result in $\left|\gamma_{p}\right|<\left|\gamma_{0}\right|$. We have also assumed that the medium is sluggish and responds only at frequencies much lower than the optical frequency $\omega_{0}$ and that the characteristic propagation time $l / c$ ( $l$ is the length of the medium, and $c$ is the speed of light) is small compared with the characteristic relaxation time $\tau$ (for most photorefractive media the latter is of the order of seconds). Equations (3) formally describe sluggish Kerr media with the replacement

$$
\frac{\left\{\gamma_{0}, \gamma_{p}\right\}}{(1+q)} \rightarrow 2 \frac{\omega_{0} n_{2}}{c}\left|F_{0}(z)\right|^{2}
$$

although the approximation of reflection gratings but no transmission gratings is not sensible in common Kerrtype media, where diffusion processes tend to preferentially wash out reflection gratings.

It is tempting to consider eliminating the direct interaction of the pump waves by arranging for them to be mutually temporally incoherent or to have orthogonal polarization states. Putting $\gamma_{p}=0$ in Eqs. (3) leads to two decoupled sets of single-sideband four-wave mixing equations:

$$
\begin{aligned}
& \left(\frac{\mathrm{d}}{\mathrm{d} z}+i k_{d}\right) F_{+1}=i \frac{q}{1+q} \chi+\gamma_{0}\left(F_{+1}+B_{-1}^{*}\right) \\
& \left(\frac{\mathrm{d}}{\mathrm{d} z}+i k_{d}\right) B_{-1}^{*}=i \frac{1}{1+q} \chi+\gamma_{0}\left(F_{+1}+B_{-1}^{*}\right) \\
& \left(\frac{\mathrm{d}}{\mathrm{d} z}-i k_{d}\right) F_{-1}^{*}=-i \frac{q}{1+q} \chi_{-}^{*} \gamma_{0}^{*}\left(F_{-1}^{*}+B_{+1}\right) \\
& \left(\frac{\mathrm{d}}{\mathrm{d} z}-i k_{d}\right) B_{+1}=-i \frac{1}{1+q} \chi{ }^{*} \gamma_{0}^{*}\left(F_{-1}^{*}+B_{+1}\right)
\end{aligned}
$$

Despite the fact that $k_{d}$ explicitly appears in Eqs. (5) and (6), they are completely decoupled, which means that they do not describe the appearance of a dual-sideband transverse instability with an explicit dependence on $k_{d}$. Analysis shows that this is true even when the mirror boundary conditions of Fig. 1(b) are used, which do couple the two sets of sidebands. This explains the experimental observation reported in Ref. 6 that the transverse instability disappears when the pump beams are made mutually temporally incoherent. In this respect the physics of the reflection-grating-mediated transverse instability is quite different from the transmission-grating case, where the instability occurs in the absence of any direct pump-pump interaction. It may be noted that Eqs. (5) and (6) are equivalent to those given in Ref. 14, which describe the on-axis stimulated-Brillouinscattering instability of counterpropagating beams. The difference between Eqs. (5) and (6) and those describing the stimulated-Brillouin-scattering case is that here the nonlinearity is proportional to the modulation depth of the field interference as opposed to being proportional to the optical intensity.

With these results in mind we therefore set $\gamma_{p}=\gamma_{0}$, which leads to the equations of motion:

$$
\begin{aligned}
\left(\frac{\mathrm{d}}{\mathrm{d} z}+i k_{d}\right) F_{+1}= & i \frac{q}{1+q} \gamma_{0}\left[\left(\chi_{+}-1\right) F_{+1}\right. \\
& \left.+B_{+1}+\chi_{+} B_{-1}^{*}\right]
\end{aligned}
$$




$$
\begin{aligned}
\left(\frac{\mathrm{d}}{\mathrm{d} z}-i k_{d}\right) F_{-1}^{*}= & -i \frac{q}{1+q} \gamma_{0}^{*}\left[\left(\chi_{-}^{*}-1\right) F_{-1}^{*}\right. \\
& \left.+\chi_{-}^{*} B_{+1}+B_{-1}^{*}\right] \\
\left(\frac{\mathrm{d}}{\mathrm{d} z}-i k_{d}\right) B_{+1}= & -i \frac{1}{1+q} \gamma_{0}^{*}\left[F_{+1}+\chi_{-}^{*} F_{-1}^{*}\right. \\
& \left.+\left(\chi_{-}^{*}-1\right) B_{+1}\right] \\
\left(\frac{\mathrm{d}}{\mathrm{d} z}+i k_{d}\right) B_{-1}^{*}= & i \frac{1}{1+q} \gamma_{0}\left[\chi_{+} F_{+1}+F_{-1}^{*}\right. \\
+(\chi+ & \left.-1) B_{-1}^{*}\right] .
\end{aligned}
$$

Analysis of the solutions of these equations constitutes the remainder of this paper.

\section{DISPERSION RELATIONS FOR REAL COUPLING CONSTANTS}

Solutions of Eqs. (7) yield dispersion relations for the threshold of the absolute instability that are functions of $q(z)$. We wish to find the value of $q(z)$ that minimizes the instability threshold. When the wave interaction is due to the formation of transmission gratings, the equations corresponding to Eqs. (7) may be solved for arbitrary values of $q(z)$ and $\gamma_{0} .^{12}$ Equations (7) do not admit of such general solutions, and we consider first the case of real $\gamma_{0}$. Solutions for complex $\gamma_{0}$ are given in Section 4 below. When $\gamma_{0}$ is real $\left(\gamma_{0}=\gamma_{0}^{\prime}\right), q(z)=$ constant, and Eqs. (7) are readily integrated in closed form. The boundary conditions $F_{+1}(0)=F_{-1}{ }^{*}(0)=B_{+1}(l)=B_{-1}{ }^{*}(l)=0$, corresponding to the geometry of Fig. 1(a), yield the following dispersion relation for the threshold of the absolute instability:

$$
\begin{aligned}
& \tilde{k}_{d}{ }^{2}\left[\chi_{+}{ }^{2}+\left(2-4 \chi_{+}+\chi_{+}{ }^{2}\right) \tilde{q}^{2}\right]+\frac{\gamma_{0}{ }^{2}}{4}\left(1-\chi_{+}\right)^{2} \tilde{q}^{2}\left(\tilde{q}^{2}-1\right) \\
& +\left(\tilde{q}^{2}-1\right)\left[-\tilde{k}_{d}{ }^{2} \chi_{+}{ }^{2}+\frac{\gamma_{0}{ }^{2}}{4}\left(1-\chi_{+}\right)^{2} \tilde{q}^{2}\right] \cos (r l) \cos (s l) \\
& +\left(1-\tilde{q}^{2}\right)\left\{\tilde{k}_{d}{ }^{4} \chi_{+}{ }^{2}+\tilde{k}_{d}{ }^{2} \frac{{\gamma_{0}}^{\prime 2}}{4}\left(1-\chi_{+}{ }^{2}\right)\left[\chi_{+}{ }^{2}+\left(1-2 \chi_{+}\right) \tilde{q}^{2}\right]\right. \\
& \left.-\frac{\gamma_{0}{ }^{4}}{16} \tilde{q}^{2}\left(1-\chi_{+}\right)^{3}\left[1+\chi_{+}\left(1-2 \tilde{q}^{2}\right)\right]\right\} \frac{\sin (r l) \sin (s l)}{r s}=0,
\end{aligned}
$$

where

$$
\begin{aligned}
r^{2}= & \tilde{k}_{d}{ }^{2}-\frac{\gamma_{0}{ }^{2}}{4}\left[1-\chi_{+}{ }^{2}-2 \tilde{q}^{2}\left(1-\chi_{+}\right)\right] \\
& +\gamma_{0}^{\prime}\left\{\tilde{k}_{d}^{2}\left[\chi_{+}^{2}+\tilde{q}^{2}\left(1-2 \chi_{+}\right)\right]\right. \\
& \left.+\frac{\gamma_{0}^{\prime 2}}{4}\left(1-\chi_{+}\right)^{2} \tilde{q}^{2}\left(\tilde{q}^{2}-1\right)\right\}^{1 / 2}, \\
s^{2}= & \tilde{k}_{d}^{2}-\frac{\gamma_{0}{ }^{2}}{4}\left[1-\chi_{+}^{2}-2 \tilde{q}^{2}\left(1-\chi_{+}\right)\right] \\
& -\gamma_{0}^{\prime}\left\{\tilde{k}_{d}^{2}\left[\chi_{+}{ }^{2}+\tilde{q}^{2}\left(1-2 \chi_{+}\right)\right]\right. \\
& \left.+\frac{\gamma_{0}^{\prime 2}}{4}\left(1-\chi_{+}\right)^{2} \tilde{q}^{2}\left(\tilde{q}^{2}-1\right)\right\}^{1 / 2},
\end{aligned}
$$

$$
\begin{aligned}
\tilde{k}_{d} & =k_{d}+\frac{\gamma_{0}{ }^{2}}{2}\left(1-\chi_{+}\right), \\
\tilde{q} & =\frac{q-1}{q+1} .
\end{aligned}
$$

These results and those that follow below were derived with $\tau$ assumed to be real such that $\chi_{-}{ }^{*}=\chi_{+}$. This is not necessary, but it simplifies the resulting formulas. The boundary conditions $F_{+1}(0)=F_{-1}^{*}(0)=0, F_{+1}(l)=B_{+1}(l)$, $F_{-1}^{*}(l)=B_{-1}{ }^{*}(l)$, corresponding to an absolute instability in the geometry of Fig. 1(b), yield the dispersion relation

$$
\begin{aligned}
& \tilde{k}_{d}^{2}\left(\frac{\tilde{q}}{\tilde{q}+1}\right)\left[\chi_{+}{ }^{2}-\left(1-2 \chi_{+}\right) \tilde{q}\right]+\left(1-\chi_{+}\right)^{2} \tilde{q}^{2} \\
& \times\left[\tilde{k}_{d} \frac{\gamma_{0}^{\prime}}{2}-\frac{\gamma_{0}^{\prime 2}}{4} \tilde{q}\right]+\left\{-\tilde{k}_{d}{ }^{2} \chi_{+}{ }^{2}+\left(1-\chi_{+}\right)^{2} \tilde{q}^{2}\right. \\
& \left.\times\left[-\tilde{k}_{d} \frac{\gamma_{0}^{\prime}}{2}+\frac{\gamma_{0}{ }^{2}}{4}\right]\right\} \cos (r l) \cos (s l) \\
& +k_{d}\left\{\tilde{k}_{d}{ }^{2} \frac{\gamma_{0}^{\prime}}{2}\left[\chi_{+}{ }^{2}\left(1+\chi_{+}\right)-\left(\chi_{+}{ }^{2}+2 \chi_{+}-1\right) \tilde{q}^{2}\right]\right. \\
& -\tilde{k}_{d} \frac{\gamma_{0}^{\prime 2}}{4} \chi_{+}\left(1-\chi_{+}\right)^{2} \tilde{q}^{2}-\frac{\gamma_{0}^{\prime 3}}{8}\left(1-\chi_{+}\right)^{2} \tilde{q}^{2}\left[1+\chi_{+}\left(1-2 \tilde{q}^{2}\right)\right] \\
& \left.-\tilde{k}_{d}{ }^{3} \chi_{+}{ }^{2}\right\} \frac{\sin (r l) \sin (s l)}{r s}=0 .
\end{aligned}
$$

The beam-intensity ratio is in this case given by $q=R$, $R$ being the mirror reflectivity.

When the counterpropagating beams have equal intensities, we have $\tilde{q}=0$, and the dispersion relations simplify to

$$
\begin{aligned}
1+\cos (r l) \cos (s l)+\left[2 \tilde{k}_{d}^{2}-\frac{1}{2}\left(r^{2}+s^{2}\right)\right] \\
\times \frac{\sin (r l) \sin (s l)}{r s}=0
\end{aligned}
$$

for external-beam boundary conditions [Fig. 1(a)] and

$$
\cos (r l) \cos (s l)+k_{d}\left(k_{d}-\gamma_{0}^{\prime} \chi_{+}\right) \frac{\sin (r l) \sin (s l)}{r s}=0
$$

for mirror boundary conditions [Fig. 1(b)]. In Eqs. (11) and (12), $r^{2}=\left(\tilde{k}_{d}+\gamma_{0}^{\prime} \chi_{+} / 2\right)^{2}-\gamma_{0}^{\prime 2} / 4$ and $s^{2}=\left(\tilde{k}_{d}-\right.$ $\left.\gamma_{0}^{\prime} \chi_{+} / 2\right)^{2}-\gamma_{0}^{\prime 2} / 4$.

The dispersion relations have an infinite number of solution branches. Consider first the external-beam case of Fig. 1(a). In the limit $k_{d} \gg\left|\gamma_{r}{ }^{0}\right|$ the two sideband gratings decouple, and Eq. (8) reduces to

$$
\begin{aligned}
\chi_{+}{ }^{2}+\tilde{q}^{2}\left(\chi_{+}{ }^{2}-\right. & \left.4 \chi_{+}+2\right)+\left(1-\tilde{q}^{2}\right) \chi_{+}{ }^{2} \\
& \times \cos \left\{\gamma_{0}^{\prime}\left[\chi_{+}{ }^{2}+\tilde{q}^{2}\left(1-2 \chi_{+}\right)\right]^{1 / 2} l\right\}=0 .
\end{aligned}
$$

Numerical solution of Eq. (13) shows that the minimum threshold occurs for $\Omega=0$, in which case Eq. (13) further reduces to

$$
1+\cos \left[2 \gamma_{0}^{\prime} \sqrt{q} /(q+1)\right]=0 .
$$

The threshold is given by $\gamma_{0}^{\prime} l=(N+1 / 2)(q+1) \pi / \sqrt{q}$, with $N$ an arbitrary integer, which for $q=1$ reduces to the well-known result for reflection-grating four-wave mixing oscillation in photorefractive media with a local 
response. ${ }^{13}$ When $q \neq 1$, Eq. (13) extends the results of Ref. 13 for the oscillation threshold by including the effect of a direct interaction between the counterpropagating pump beams. The dependence of the threshold on $q$ as given by Eq. (14) is shown in Fig. 2. To compare the present results with those of Ref. 13, we have used $\gamma=\gamma_{0}^{\prime} /(1-i \Omega \tau)$ in the threshold condition $\cosh [-i \gamma l / 2+\ln (q) / 2]=0$ [Eq. (2.15) of Ref. 13]. We see that the direct interaction of the counterpropagating pump beams tends to raise the oscillation threshold as measured by $\left|\gamma_{0}^{\prime}\right|$. There is also a qualitative difference when the effect of the pump-pump grating is included. When the pump-pump grating is absent, four-wave mixing oscillation in the presence of unequal-intensity pump beams is possible only with detuned sidebands. When the pump-pump grating is included, there is no frequency shift of the sidebands even with unequal pump beams. The coincidence of the threshold condition for $q=1$ calculated here with that calculated in Ref. 13 is somewhat fortuitous and is related to the fact that for $q=1$ there is reflection symmetry about the line $z=l / 2$.

We have plotted the lowest-lying solution branches of Eq. (8) in Fig. 3 by finding the values of $\gamma_{0}^{\prime} l$ and $\Omega \tau$ that minimize $\left|\gamma_{0}^{\prime} l\right|$ while satisfying the dispersion relation for each value of $k_{d} l$. The minimum threshold of $\left|\gamma_{0}^{\prime} l\right| \sim 2.1$ occurs for $q=1$, in which case the focusing $\left(\gamma_{0}^{\prime}>0\right)$ and defocusing $\left(\gamma_{0}^{\prime}<0\right)$ branches are equivalent: changing the sign of $\gamma_{0}^{\prime}$ corresponds to relabeling the positive $\hat{z}$ direction. The scalloped form of the threshold curves arises because the dispersion relation factors into a product of terms, ${ }^{10,12}$ leading to several solutions for each value of $k_{d} l$. For $q=1$, factorization of Eq. (11) gives

$$
\begin{aligned}
{[\tan (r l / 2) \tan (s l / 2)+} & \left.a_{1}\right]\left[\tan (r l / 2) \tan (s l / 2)+a_{2}\right] \\
& \times\left[\tan (r l / 2) \tan (s l / 2)+1 / a_{1}\right] \\
& \times\left[\tan (r l / 2) \tan (s l / 2)+1 / a_{2}\right]=0,
\end{aligned}
$$

$$
a_{1,2}=\left[\left(\tilde{k}_{d} \pm \gamma_{0}^{\prime} / 2\right)^{2}-\frac{\gamma_{0}^{\prime 2}}{4} \chi_{+}^{2}\right] l^{2}
$$

Only the lowest of the solutions is shown in Fig. 3, while the sharp cusps correspond to points where the solution branches cross. For $q \neq 1$ and $k_{d} l \geqslant 2$ the lowest threshold occurs for $\Omega=0$, in which case Eq. (8) factors into the form

$$
[\tan (r l / 2) \tan (s l / 2)+r / s][\tan (r l / 2) \tan (s l / 2)+s / r]=0,
$$

$$
\begin{aligned}
& r^{2}=k_{d}^{2}+k_{d} \gamma_{0}^{\prime}\left(1-\tilde{q}^{2}\right)^{1 / 2}, \\
& s^{2}=k_{d}{ }^{2}-k_{d} \gamma_{0}^{\prime}\left(1-\tilde{q}^{2}\right)^{1 / 2}
\end{aligned}
$$

Thus, when the lowest threshold occurs for $\Omega=0$, the dispersion curves with $q \neq 1$ are identical to those with $q=1$ but with $\gamma_{0}^{\prime}$ scaled by $1 /\left(1-\tilde{q}^{2}\right)^{1 / 2}$. For $q \neq 1$ and small $k_{d} l$ the focusing branches have $\Omega \neq 0$, whereas the defocusing branches always have $\Omega=0$. Additional calculations, not plotted in Fig. 3, show that as $q$ increases the value of $k_{d} l$ at which the focusing branch becomes detuned increases. This is complementary to the transmission grating case, ${ }^{12}$ for which the value of $k_{d} l$ at which the focusing branch detunes decreases with increasing $q$.

For mirror boundary conditions [Fig. 1(b)] the limit of $k_{d} \gg\left|\gamma_{0}^{\prime}\right|$ gives

$$
\begin{aligned}
\tilde{q} \chi_{+}{ }^{2}-\tilde{q}^{2}(1- & \left.2 \chi_{+}\right)-\chi_{+}{ }^{2}(1+\tilde{q}) \\
& \times \cos \left\{\gamma_{0}^{\prime}\left[\chi_{+}{ }^{2}+\tilde{q}^{2}\left(1-2 \chi_{+}\right)\right]^{1 / 2} l\right\}=0
\end{aligned}
$$

for the dispersion relation. When the pump beams are of equal intensity, Eq. (17) reduces to $\cos \left(\gamma_{0}^{\prime} \chi+l\right)=0$. The minimum threshold is thus $\gamma_{0}^{\prime} l=\pi / 2$ and $\Omega=0$, which is half the minimum threshold without mirror boundary conditions. This result may be attributed to the mirror's feeding energy from the sidebands back into the interaction region. The instability thresholds calculated from Eq. (10) for $q$ equal to $1,1 / 5$, and $1 / 25$ are shown in Fig. 4. The absolute minimum threshold of $\left|\gamma_{0}^{\prime} l\right| \sim 1.1$ is found on the focusing side with $q=1$ at $k_{d} l \sim 1.4$ and is roughly a factor of 2 lower than the minimum threshold without a mirror. The presence of the mirror breaks the reflection symmetry about $z=l / 2$, so even for $q=1$ the

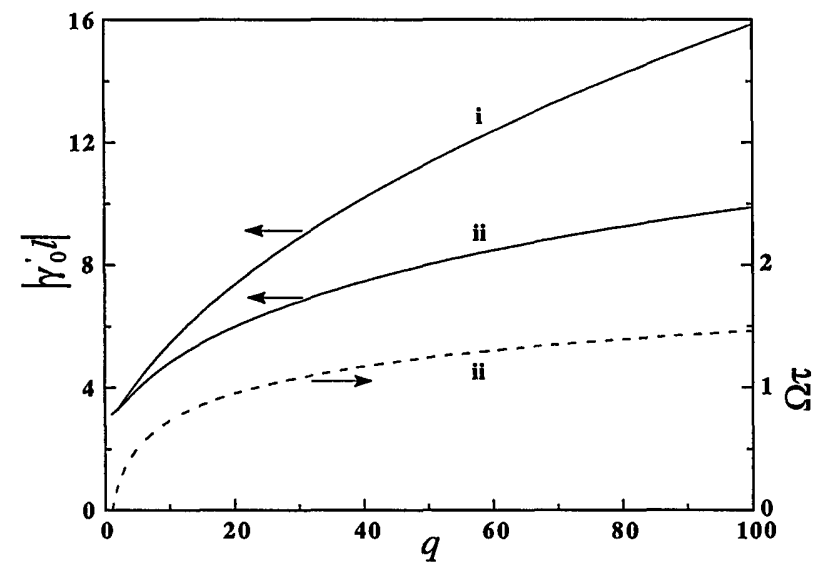

Fig. 2. Four-wave mixing oscillation threshold as a function of the pump-beam intensity ratio $q$. Curve $i$ is calculated from Eq. (14) and curves ii from the theory of Ref. 13. Solid curves, $\left|\gamma_{0}{ }^{\prime} l\right|$; dashed curve, $\Omega \tau$.

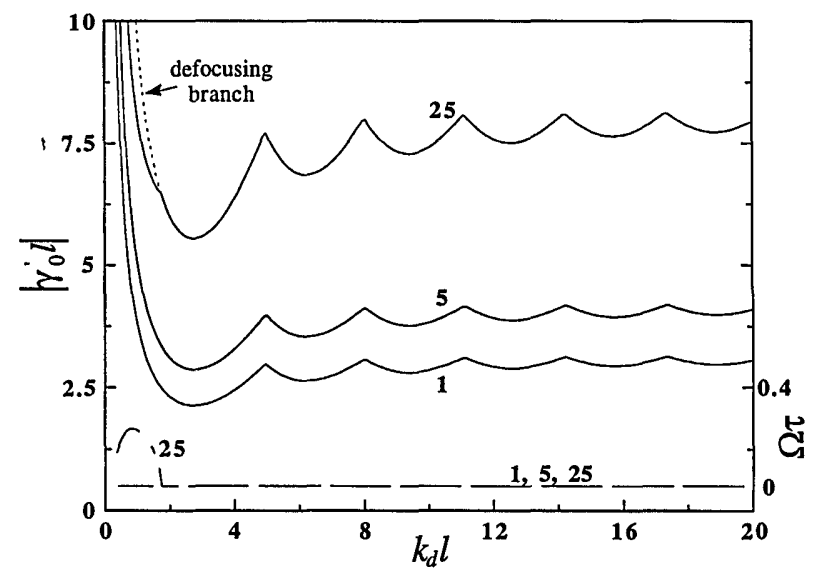

Fig. 3. Instability thresholds for real $\gamma_{0}=\gamma_{0}^{\prime}$ and externally supplied beams. The curves are labeled with the value of $q$, and $\Omega \tau$ is identically zero for all branches except for the $q=25$ focusing branch. The focusing $\left(\gamma_{0}^{\prime}>0\right)$ and defocusing $\left(\gamma_{0}^{\prime}<0\right)$ branches for a given value of $q$ coincide, except where $\Omega \tau \neq 0$. Solid curves $\left|\gamma_{0}{ }^{\prime} l\right|$; long-dashed curves, $\Omega \tau$. 


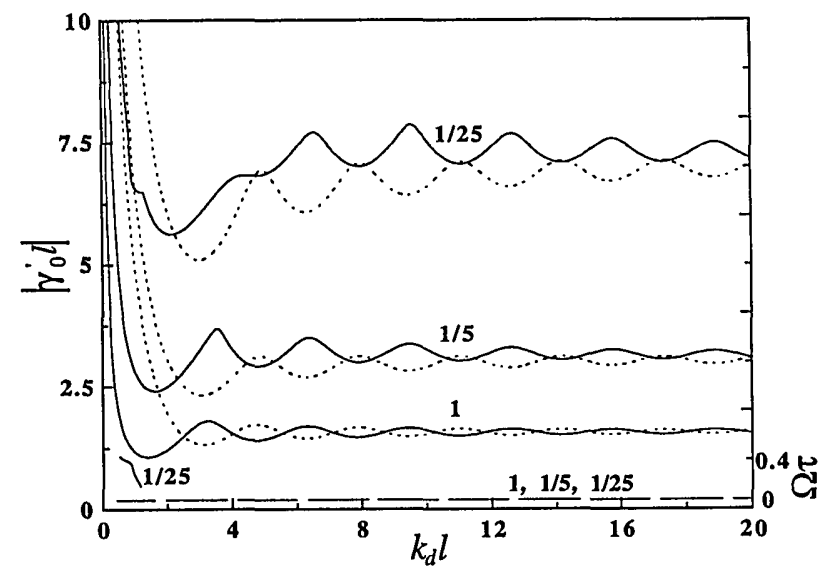

Fig. 4. Instability thresholds for real $\gamma_{0}=\gamma_{0}{ }^{\prime}$ and mirror boundary conditions. The curves are labeled with the value of $q$, and $\Omega \tau$ is identically zero for all branches except for the $q=1 / 25$ focusing branch. Solid curves, focusing branches; dotted curves, defocusing branches; long-dashed curves, $\Omega \tau$.

focusing and the defocusing branches are not equivalent. As the pump-beam intensities become more unequal, the intertwined focusing and defocusing branches move apart from each other, with the defocusing branches having the lowest threshold. Referring to Eqs. (1), we see that this corresponds physically to the case in which the phases of $F_{0}$ and $B_{0}$ are minimized at the mirror.

\section{DISPERSION RELATIONS FOR COMPLEX COUPLING CONSTANTS}

When the coupling constant has a finite imaginary part, the counterpropagating pump beams exchange energy, and $q(z)$ becomes position dependent, complicating the analysis of Eqs. (7). The exact conditions used in the experimental observation of hexagons in photorefractive media $^{6}$ are therefore not readily analyzed. However, even when the counterpropagating beams exchange energy it is possible to obtain $q(z)=1$ by correct choice of the boundary conditions. The case $q(z)=1$ is also of interest because it probably represents the minimum instability threshold. ${ }^{15}$ This is certainly the case when $\gamma_{0}$ is purely real, as was shown in Section 3 above, and also for the transmission grating case with an arbitrary complex coupling constant ${ }^{12}$ or for combined transmission and reflection gratings with a real coupling constant. ${ }^{10}$ Note also that the solutions of Eqs. (7) remain unchanged if we make the replacement $q \rightarrow 1 / q$; since the physical situation is identical, we have merely renamed the forward and the backward propagating waves. It follows that $q(z)=1$ is an extremum of the threshold condition with respect to variations in $q$. We argue that this extremum is in fact the minimum both by analogy with the known results cited above and physically, because the strength of the induced change in the refractive index of the medium is proportional to $\sqrt{q} /(1+q)$, which has a maximum at $q=1$.

To obtain $q(z)=1$, note that all solutions of Eqs. (1b) satisfy $\left|F_{0}(z)\right|^{2}-\left|B_{0}(z)\right|^{2}=$ constant. The constant is zero, giving $q(z)=1$, for $\left|F_{0}(0)\right|^{2} /\left|B_{0}(l)\right|^{2}=\exp \left(\gamma_{0}^{\prime \prime} l\right)$, where the complex coupling coefficient has been written as $\gamma_{0}=\gamma_{0}^{\prime}+i \gamma_{0}^{\prime \prime}$. Alternatively, using the geometry of Fig. 1(b), the constant is identically zero independent of the coupling constant for a perfectly reflecting surface at $z=l$. It should be emphasized that even though $q(z)=1$ inside the photorefractive medium there is large variation in the pump-beam intensities along $z$ owing to the strong coupling of energy between the counterpropagating pump beams, and the externally supplied beams have unequal intensities at the two ends of the medium.

Solving Eqs. (7) with $q=1$ and external-beam boundary conditions yields

$$
\begin{aligned}
& \cosh \left(\gamma_{0}^{\prime \prime} \chi_{+} l\right)+\cos (r l) \cos (s l) \\
& +\left[2 \tilde{k}_{d}^{2}-\frac{1}{2}\left(r^{2}+s^{2}\right)\right] \frac{\sin (r l) \sin (s l)}{r s}=0
\end{aligned}
$$

for the dispersion relation. Solving Eqs. (7) with $q=1$ and mirror boundary conditions yields

$$
\begin{gathered}
\cos (r l) \cos (s l)+\frac{\gamma_{0}^{\prime \prime}}{2}\left[\cos (r l) \frac{\sin (s l)}{s}+\frac{\sin (r l)}{r} \cos (s l)\right] \\
+\left[k_{d}\left(k_{d}-\gamma_{0}^{\prime} \chi_{+}\right)+\frac{\gamma_{0}^{\prime \prime 2}}{4}\right] \frac{\sin (r l) \sin (s l)}{r s}=0
\end{gathered}
$$

for the dispersion relation. In Eqs. (18) and (19) $r^{2}=$ $\left(\tilde{k}_{d}+\gamma_{0}^{\prime} \chi_{+} / 2\right)^{2}-\left|\gamma_{0}\right|^{2} / 4$ and $s^{2}=\left(\tilde{k}_{d}-\gamma_{0}^{\prime} \chi_{+} / 2\right)^{2}-$ $\left|\gamma_{0}\right|^{2} / 4$. Equation (18) is independent of the sign of $\gamma_{0}^{\prime \prime}$, whereas the dispersion relation (19) for the case of mirror boundary conditions does depend on the sign of $\gamma_{0}^{\prime \prime}$. For $\gamma_{0}^{\prime \prime}>0$ the optical intensity reaches its minimum at the mirror, and for $\gamma_{0}^{\prime \prime}<0$ the optical intensity is a maximum at the mirror; these are physically distinct configurations. Note also that when $\gamma_{0}^{\prime}=0$ then Eq. (19) has no dependence on $\chi_{+}$and in fact no solutions. Mirror boundary conditions are therefore of limited utility when $\gamma_{0}$ is almost purely imaginary.

Equations (18) and (19) have an infinite number of solution branches. In the limiting case of $k_{d} \gg\left|\gamma_{0}\right|$ Eq. (18) reduces to

$$
\cos \left(\gamma_{0}^{\prime} \chi+l\right)+\cosh \left(\gamma_{0}^{\prime \prime} \chi+l\right)=0
$$

The lowest branch has a minimum threshold $\gamma_{0}=\pi$ and $\Omega=0$, which was determined in Section 3 . The dependence of the four-wave mixing threshold on $\arg \left(\gamma_{0}\right)$ is shown in Fig. 5. The theory of Ref. 13 when evaluated for $q=1$ yields the same threshold coupling and detuning as shown in Fig. 5 except that $\Omega<0$ when $\arg \left(\gamma_{0}\right)$ is in the first or the third quadrants. The dispersion relation for mirror boundary conditions, Eq. (19), reduces to

$$
\cos \left(\gamma_{0}^{\prime} \chi+l\right)=0
$$

with $\gamma_{0}^{\prime \prime}$ arbitrary. The large $k_{d}$ threshold with mirror boundary conditions is thus independent of $\arg \left(\gamma_{0}\right)$ provided that $\gamma_{0}^{\prime} \neq 0$.

To evaluate the instability threshold with varying degrees of energy coupling, we numerically solve the dispersion relations with $\arg \left(\gamma_{0}\right)$ fixed while allowing $\left|\gamma_{0}\right|$ and $\Omega$ to vary. The lowest-lying branches with external-beam boundary conditions are shown in Fig. 6(a) for $\left|\gamma_{0}^{\prime}\right|=\left|\gamma_{0}^{\prime \prime}\right|$, that is, $\arg \left(\gamma_{0}\right)=\pi / 4$ and $\arg \left(\gamma_{0}\right)=3 \pi / 4$, and in Fig. 6(b) for $\arg \left(\gamma_{0}\right)=\pi / 2$. The threshold curves for $\arg \left(\gamma_{0}\right)=$ $5 \pi / 4,7 \pi / 4$ are not shown, since they coincide with the curves for $\arg \left(\gamma_{0}\right)=3 \pi / 4, \pi / 4$, respectively. This is a 


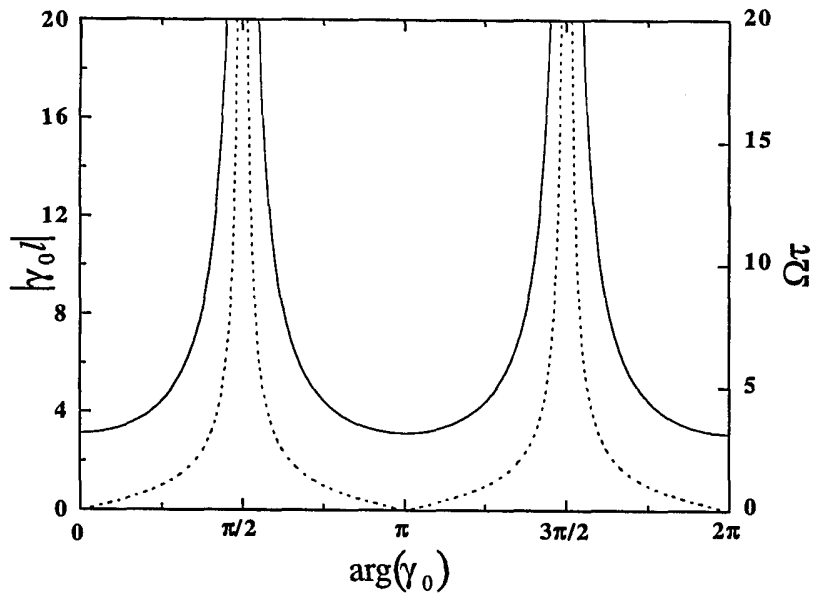

Fig. 5. Four-wave mixing oscillation threshold as a function of $\arg \left(\gamma_{0}\right)$ for $q=1$. Solid curve, $\left|\gamma_{0} l\right|$; dotted curve, $\Omega \tau$.

consequence of Eq. (18), which is independent of the sign of $\gamma_{0}^{\prime \prime}$. For the worst case of $\arg \left(\gamma_{0}\right)=\pi / 2$ the minimum threshold is increased to $\left|\gamma_{0} l\right| \sim 6.3$ as compared with $\left|\gamma_{0} l\right| \sim 2.1$ for $\arg \left(\gamma_{0}\right)=0$. Corresponding results for mirror boundary conditions are shown in Fig. 7 for $\arg \left(\gamma_{0}\right)=\pi / 4,3 \pi / 4,5 \pi / 4,7 \pi / 4$. With mirror boundary conditions some of the solutions with energy coupling $\left[\arg \left(\gamma_{0}\right)=7 \pi / 4\right]$ actually have lower minimum thresholds than solutions without energy coupling $\left[\arg \left(\gamma_{0}\right)=\pi\right]$, although the absolute minimum occurs for no energy coupling. Note also that all the curves shown in Fig. 7 have $\Omega \tau=0$ and are thus qualitatively different than the external-beam thresholds.

When the real part of $\gamma_{0}$ is nonzero, mirror boundary conditions generally give the lowest threshold, whereas for a purely imaginary $\gamma_{0}$ instability is possible only with external-beam boundary conditions. In the photorefractive media to be discussed in the following section the reflection-grating coupling constant is almost purely imaginary. To determine which type of boundary conditions are preferable in this situation, we plot the minimum value of $\left|\gamma_{0}\right|$ as a function of $\arg \left(\gamma_{0}\right)$ in Fig. 8. The crossover point between external-beam and mirror boundary conditions occurs at $\arg \left(\gamma_{0}\right) \sim \pi / 2-0.08$. Since most photorefractive media in the reflection-grating geometry have $\arg \left(\gamma_{0}\right)$ closer to $\pi / 2$ than this, externalbeam boundary conditions will typically be preferable.

\section{PHOTOREFRACTIVE MEDIA}

We turn now to a comparison of several characteristic types of photorefractive medium. The coupling coefficient describing the interaction of counterpropagating optical waves traveling along the symmetry axis of a photorefractive crystal is

$$
\gamma_{\mathrm{pr}}\left(\mathbf{k}_{g}, \Omega\right)=\left(\frac{\omega_{0} n_{0}^{3} r_{13}}{2 c}\right) E_{\mathrm{sc}}\left(\mathbf{k}_{g}\right)
$$

where $\mathbf{k}_{g}=2 k_{0} \hat{z}$. The coupling coefficient is due to photoinduced charge redistribution that results in a spacecharge field that modifies the index of refraction by the Pockels effect. Here $c$ is the speed of light in vacuum, $n_{0}$ is the ordinary index of refraction, $r_{13}$ is the relevant component of the electro-optic tensor, and $E_{\mathrm{sc}}\left(\mathbf{k}_{g}\right)$ is the photoinduced space-charge field along $\mathbf{k}_{g}$. The phase of $\gamma_{\mathrm{pr}}$ is determined by the space-charge field, which is given by $^{16}$

$$
E_{\mathrm{sc}}\left(\mathbf{k}_{g}, \Omega\right)=E_{\mathrm{sc}}{ }^{0}\left(\mathbf{k}_{\mathrm{g}}\right) \frac{1}{1-i \Omega \tau\left(\mathbf{k}_{g}\right)},
$$

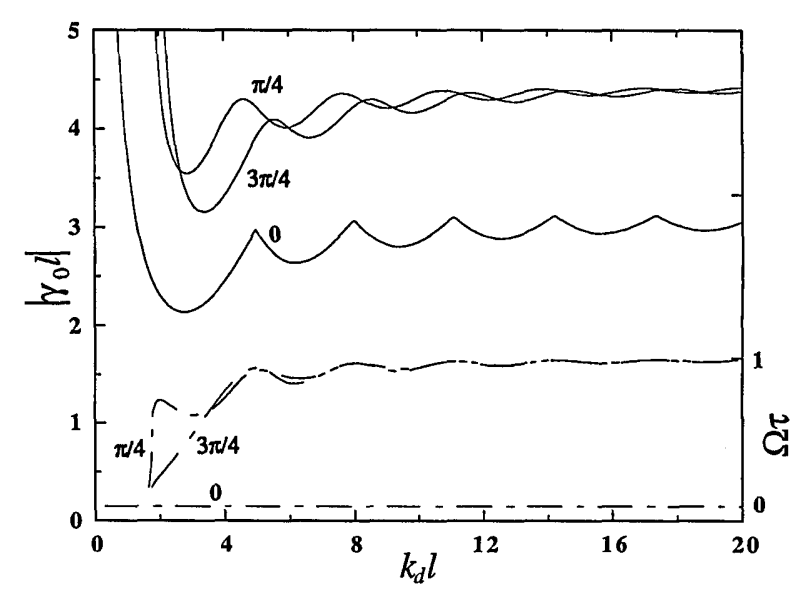

(a)

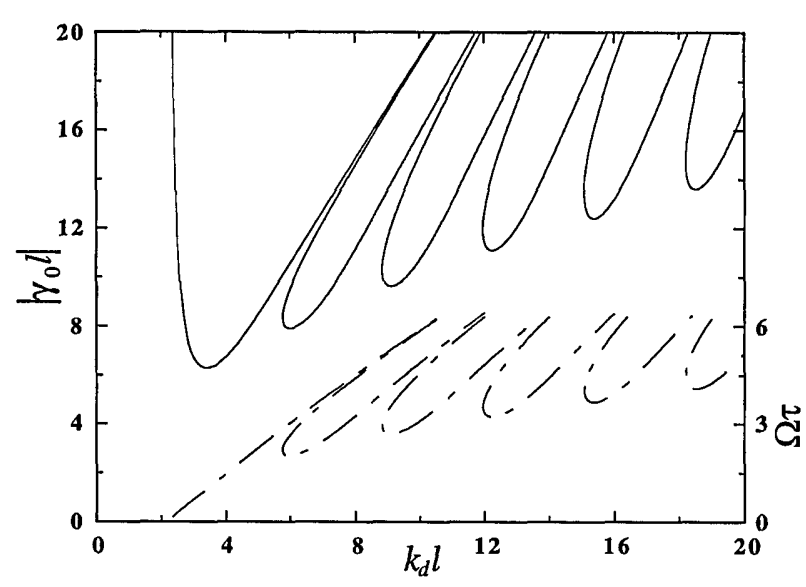

(b)

Fig. 6. Instability thresholds with external beams and $q=1$ : (a) $\arg \left(\gamma_{0}\right)=0, \pi / 4,3 \pi / 4$; (b) $\arg \left(\gamma_{0}\right)=\pi / 2$. In (a) the curves are labeled with the value of $\arg \left(\gamma_{0}\right)$. Solid curves, $\left|\gamma_{0} l\right|$; dashed curves, $\Omega \tau$.

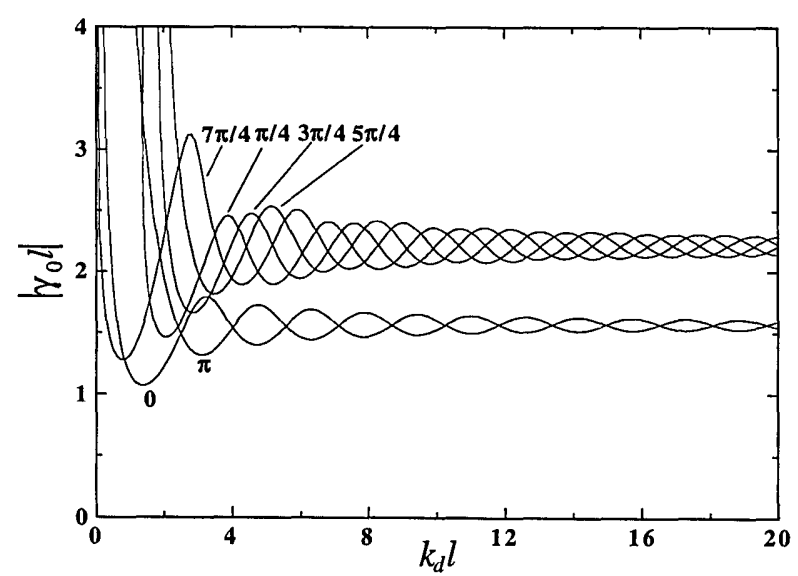

Fig. 7. Instability thresholds with mirror boundary conditions and $q=1$. The curves are labeled with the value of $\arg \left(\gamma_{0}\right)$, and $\Omega \tau=0$ for all the curves. 


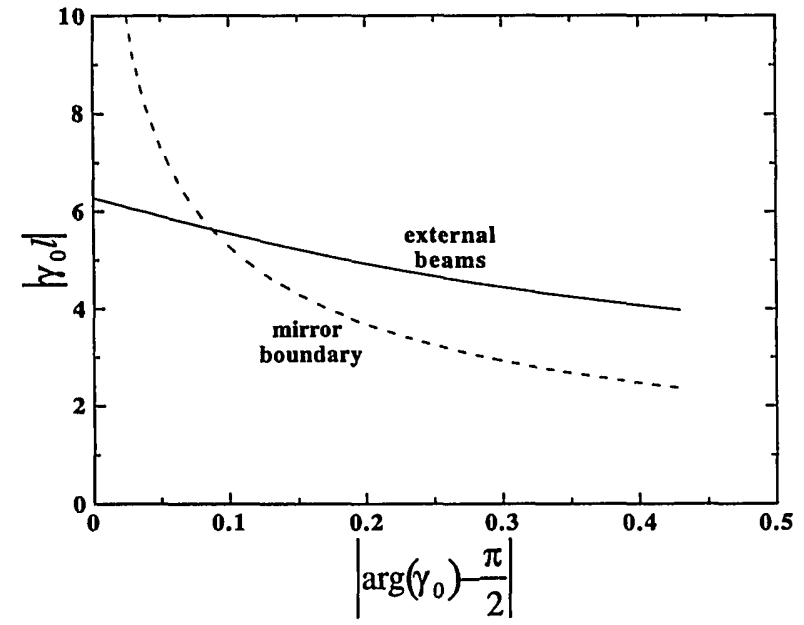

Fig. 8. Absolute minimum instability thresholds as a function of $\arg \left(\gamma_{0}\right)$ for external-beam and mirror boundary conditions.

with

$$
\begin{aligned}
E_{\mathrm{sc}}{ }^{0}\left(\mathbf{k}_{g}\right) & =E_{\max }\left(\mathbf{k}_{\mathrm{g}}\right) \frac{E_{0}\left(\mathbf{k}_{g}\right)+i E_{\mathrm{diff}}\left(\mathbf{k}_{g}\right)}{E_{\max }\left(\mathbf{k}_{g}\right)+E_{\mathrm{diff}}\left(\mathbf{k}_{g}\right)-i E_{0}}, \\
\tau\left(\mathbf{k}_{g}\right) & =t_{0} \frac{E_{\mu}\left(\mathbf{k}_{g}\right)+E_{\mathrm{diff}}\left(\mathbf{k}_{g}\right)-i E_{0}}{E_{\max }\left(\mathbf{k}_{g}\right)+E_{\mathrm{diff}}\left(\mathbf{k}_{g}\right)-i E_{0}}, \\
E_{0} & =E_{\mathrm{app}}+E_{\mathrm{pv}} .
\end{aligned}
$$

The space-charge field is expressed in terms of a number of characteristic fields: $E_{\max }=e N / \epsilon_{3} k_{g}$, the limiting space-charge field ( $e$ is the electronic charge, $N$ is the density of traps, and $\epsilon_{3}$ is the third component of the dc dielectric tensor); $E_{\text {diff }}=k_{B} T k_{g} / e$, the diffusion field ( $k_{B}$ is Boltzmann's constant, and $T$ is the absolute temperature); $E_{\mu}=\gamma_{D} N / \mu k_{g}$, the drift field ( $\gamma_{D}$ is the recombination rate of charge carriers, and $\mu$ is the mobility); and $E_{0}$, which is due to the combined effect of the photovoltaic field $E_{\mathrm{pv}}$ and any external field $E_{\mathrm{app}}$ applied along the $c$ axis. We use a simple model for the photovoltaic effect such that $E_{\mathrm{pv}}$ is the local part of the photovoltaic field directed along the $c$ axis $^{17}$ and is assumed to have no dependence on $\mathbf{k}_{g}$. The time constant $\tau$ scales with the characteristic material response time $t_{0}$, which is inversely proportional to the optical intensity. The values of all these parameters may vary considerably between different samples of any particular material. The values given in Table 1 are meant to be representative; see e.g., Ref. 18 for references to the original literature.
The coupling and time constants for $\mathrm{BaTiO}_{3}, \mathrm{LiNbO}_{3}$, and $\mathrm{KNbO}_{3}$ are shown in the last three lines of Table 1. When the interaction is due to the formation of transmission gratings with correspondingly small values for $k_{g}, \mathrm{BaTiO}_{3}$ and $\mathrm{LiNbO}_{3}$ have quite different properties; $\mathrm{BaTiO}_{3}$ is diffusion dominated, leading to $\arg \left(\gamma_{0}\right) \sim \pi / 2$, whereas $\mathrm{LiNbO}_{3}$ is drift dominated, leading to $\arg \left(\gamma_{0}\right) \sim 0$. However, in the reflection grating case, for which $k_{g}$ is large compared with the Debye screening wave vector, the photorefractive materials considered here are all diffusion dominated and hence have similar characteristics. The most significant difference between $\mathrm{BaTiO}_{3}, \mathrm{LiNbO}_{3}$, and $\mathrm{KNbO}_{3}$ in this configuration is simply the magnitude of $\gamma_{0}$. Equal trap densities in all three materials were assumed in Table 1 for the sake of comparison, which leads to the conclusion that $\mathrm{KNbO}_{3}$ is the preferred material. In practice trap densities vary considerably between samples, and any of the three materials examined here could be suitable for observation of instabilities.

In $\mathrm{BaTiO}_{3}$ and $\mathrm{KNbO}_{3} \arg \left(\gamma_{0}\right)$ is close to $\pi / 2$, so external-beam boundary conditions give the lowest threshold. For $\mathrm{LiNbO}_{3}$ the value of $\arg \left(\gamma_{0}\right)$ is very close to the crossover point in Fig. 8, so either type of boundary condition should give similar thresholds. Note, however, that the value of $\arg \left(\gamma_{0}\right)$ in $\mathrm{LiNbO}_{3}$ is strongly dependent on the value of the photovoltaic field, which may vary by 4 orders of magnitude in $\mathrm{LiNbO}_{3}$. In samples with large photovoltaic fields $\arg \left(\gamma_{0}\right)$ will decrease, and mirror boundary conditions are to be preferred. On the other hand, samples of $\mathrm{LiNbO}_{3}$ in which the photovoltaic field is strong are known to exhibit strong fanning and may, on those grounds alone, be unsuitable for the observation of transverse instabilities. Using the calculated coupling constant values from Table 1 for external-beam boundary conditions implies that a nonlinear medium length of 1-2 cm should be sufficient for experimental observation of the instability.

It is possible to make a rough comparison with the experimental observations of Ref. 6, which were based on illumination of a 5-mm-long sample of $\mathrm{KNbO}_{3}$ with unequal-intensity beams. The experimental conditions did not correspond exactly to any of the calculations reported here, since the externally supplied beams were not of the correct intensity to make the beam-intensity ratio independent of position. Apart from the internal variation of the beam-intensity ratio, the calculations of Fig. 6(b) correspond fairly closely to the experimental

Table 1. Physical Parameters of Some Photorefractive Crystals ${ }^{a}$

\begin{tabular}{lcccc}
\hline \multicolumn{1}{c}{ Parameter } & Symbol & $\mathrm{BaTiO}_{3}$ & $\mathrm{LiNbO}_{3}$ & $\mathrm{KNbO}_{3}$ \\
\hline Refractive index & $n_{0}$ & 2.46 & 2.34 & 2.23 \\
Dc dielectric constant & $\epsilon_{3}$ & 106 & 29 & 55 \\
Electro-optic coefficient $(\mathrm{pm} / \mathrm{V})$ & $r_{13}$ & 19.5 & 8.6 & 28 \\
Photovoltaic field $(\mathrm{V} / \mathrm{cm})$ & $E_{\mathrm{pv}}$ & $10 . \times 10^{4}$ & $1 . \times 10^{3}$ \\
Trap density $\left(\mathrm{m}^{-3}\right)$ & $N$ & $4 . \times 10^{22}$ & $4 . \times 10^{22}$ & $4 . \times 10^{22}$ \\
Recombination rate $\left(\mathrm{m}^{3} / \mathrm{s}\right)$ & $\gamma D$ & $5 . \times 10^{-14}$ & $5 . \times 10^{-14}$ & $5 . \times 10^{-16}$ \\
Mobility $\left(\mathrm{m}^{2} / \mathrm{V} \mathrm{s}\right)$ & $\mu$ & $5 . \times 10^{-5}$ & $8 . \times 10^{-5}$ & $5 . \times 10^{-5}$ \\
Magnitude of coupling constant $\left(\mathrm{cm}^{-1}\right)$ & $\mid \gamma_{0}$ & 1.9 & 2.4 & 3.9 \\
Argument of coupling constant & $\arg \left(\gamma_{0}\right)$ & 1.57 & 1.45 & 1.56 \\
Argument of time constant & $\arg (\tau)$ & $1.5 \times 10^{-4}$ & $8.0 \times 10^{-4}$ & $-1.0 \times 10^{-3}$ \\
\hline
\end{tabular}

${ }^{a}$ The last three rows are calculated for 514-nm laser light and reflection gratings with $\mathrm{k}_{\mathrm{g}}$ along the $z$ axis. 
situation. Translating the results to the experimental conditions, Fig. 6 (b) predicts a minimum-intensity coupling constant of $\left|2 \gamma_{0} l\right| \sim 12.6$ and an external angle between the primary beam and the satellites of $0.9^{\circ}$. The values reported in Ref. 6 were $\left|2 \gamma_{0} l\right| \sim 6.3$ and an external angle of $0.9-1.1^{\circ}$. While the predicted angle is in good agreement with the experiment, the value of the coupling constant is not. The source of the discrepancy is unknown.

To recapitulate, the transverse instability of counterpropagating beams in photorefractive media that is due to the formation of reflection gratings has been studied. We obtained analytic solutions including the effect of strong energy coupling between the primary waves by fixing the boundary conditions such that the ratio of the counterpropagating intensities inside the medium remains constant. This implies that when the coupling constant is complex the externally supplied beams should have unequal intensities. The calculated minimum thresholds correspond, using commonly available photorefractive media, to a nonlinear medium thickness of the order of $1 \mathrm{~cm}$, and the generated satellite beams are predicted to be detuned from the pump beams unless mirror boundary conditions are used.

\section{ACKNOWLEDGMENTS}

This work was supported by National Science Foundation (NSF) grant PHY 90-12244, U.S. Air Force Office of Scientific Research (AFOSR) grant AFOSR-90-0198, U.S. Office of Naval Research grant N00014-88-K-0083, and the Optoelectronic Computing Systems Center, a NSF Engineering Research Center. M. Saffman thanks the AFOSR for a laboratory graduate fellowship.

\section{REFERENCES AND NOTES}

1. G. Grynberg, E. Le Bihan, P. Verkerk, P. Simoneau, J. R. R. Leite, D. Bloch, S. Le Boiteux, and M. Ducloy, "Observation of instabilities due to mirrorless four-wave mixing oscillation in sodium," Opt. Commun. 67, 363-366 (1988).

2. A. Petrossian, M. Pinard, A. Maître, J.-Y. Courtois, and G. Grynberg, "Transverse-pattern formation for counterpropagating beams in rubidium vapor," Europhys. Lett. 18, 689-695 (1992).

3. J. Pender and L. Hesselink, "Conical emissions and phase conjugation in atomic sodium vapor," IEEE J. Quantum Electron. 25, 395-402 (1989); "Degenerate conical emission in atomic-sodium vapor," J. Opt. Soc. Am. B 7, 1361-1373 (1990).

4. R. Macdonald and H. J. Eichler, "Spontaneous optical pattern formation in a nematic liquid crystal with feedback mirror," Opt. Commun. 89, 289-295 (1992).
5. M. Tamburrini, M. Bonavita, S. Wabnitz, and E. Santamato, "Hexagonally patterned beam filamentation in a thin liquidcrystal film with a single feedback mirror," Opt. Lett. 18, 855-857 (1993).

6. T. Honda, "Hexagonal pattern formation due to counterpropagation in $\mathrm{KNbO}_{3}$," Opt. Lett. 18, 598-600 (1993).

7. S. N. Vlasov and V. I. Talanov, "About some features of scattering of signal wave on counterpropagating pump beams under conditions of degenerate four-photon interaction," in Optical Phase Conjugation in Nonlinear Media, V. I. Bespalov, ed. (Institute of Applied Physics, USSR Academy of Sciences, Gorki, 1979), pp. 85-91; S. N. Vlasov and E. V. Sheinina, "Theory of the interaction of waves traveling in opposite directions in a nonlinear cubic medium," Izv. Vyssh. Ucebn. Zaved. Radiofiz. 26, 20-28 (1983) [Radiophys. Quantum Electron. 27, 15-22 (1983)].

8. W. J. Firth and C. Paré, "Transverse modulational instabilities for counterpropagating beams in Kerr media," Opt. Lett. 13, 1096-1098 (1988).

9. G. Grynberg and J. Paye, "Spatial instability for a standing wave in a nonlinear medium," Europhys. Lett. 8, 29-33 (1989).

10. W. J. Firth, A. Fitzgerald, and C. Paré, "Transverse instabilities due to counterpropagation in Kerr media," J. Opt. Soc. Am. B 7, 1087-1097 (1990).

11. G. G. Luther and C. J. McKinstrie, "Transverse modulational instability of collinear waves," J. Opt. Soc. Am. B 7, 1125-1141 (1990); "Transverse modulational instability of counterpropagating light waves," J. Opt. Soc. Am. B 9, 1047-1060 (1992).

12. M. Saffman, D. Montgomery, A. A. Zozulya, K. Kuroda, and D. Z. Anderson, "Transverse instability of counterpropagating waves in photorefractive media," Phys. Rev. A 48, 3209-3215 (1993).

13. M. Cronin-Golomb, B. Fischer, J. O. White, and A. Yariv, "Theory and applications of four-wave mixing in photorefractive media," IEEE J. Quantum Electron. 20, 12-30 (1984).

14. B. Ya. Zel'dovich and V. V. Shkunov, "Characteristics of stimulated scattering in opposite pump beams," Kvantovaya Elektron. 9, 393-395 (1982) [Sov. J. Quantum Electron. 12, $223-225$ (1982)].

15. Note, however, that in the stimulated Brillouin scattering of counterpropagating beams discussed in Ref. 14 the oscillation threshold is a minimum when the pump beams have unequal intensities.

16. N. V. Kukhtarev, V. B. Markov, S. G. Odulov, M. S. Soskin, and V. L. Vinetskii, "Holographic storage in electrooptic crystals. I. Steady state," Ferroelectrics 22, 949-960 (1979).

17. B. Sturman, "The photogalvanic effect-a new mechanism of nonlinear wave interaction in electrooptic crystals," Kvantovaya Elektron. 7, 483-485 (1980) [Sov. J. Quantum Electron. 10, 276-278 (1980)].

18. P. Günter and J.-P. Huignard, eds., Photorefractive Materials and Their Applications I (Springer-Verlag, Berlin, 1988); M. P. Petrov, S. I. Stepanov, and A. V. Khomenko, Photorefractive Crystals in Coherent Optical Systems (SpringerVerlag, Berlin, 1991). 